\title{
Identifying myocardial injuries in "normal-appearing" myocardium in pediatric patients with clinically suspected myocarditis using mapping techniques
}

\author{
Haipeng Wang ${ }^{1}$, Bin Zhao ${ }^{2}$, Huan Yang ${ }^{1}$, Tianyi Qian ${ }^{3}$, Bo Han ${ }^{4}$, Haipeng Jia ${ }^{5}$, Jing An ${ }^{6}$, Junyu Zhao ${ }^{7}$, Ximing \\ Wang $^{1}$, Cuiyan Wang ${ }^{\text {Corresp. } 2}$ \\ ${ }^{1}$ Department of Radiology, Shandong Provincial Hospital affiliated to Shandong First Medical University, Ji'nan, China \\ Shandong Medical Imaging Research Institute Affiliated to Shandong University, Ji'nan, China \\ 3 Siemens Healthcare, MR Collaborations NE Asia, Beijing, China \\ 4 Department of Pediatrics, Shandong Provincial Hospital affiliated to Shandong First Medical University, Ji'nan, China \\ 5 Department of Radiology, Qilu Hospital of Shandong University, Ji'nan, China \\ 6 Siemens SSMR, APPL, Beijing, China \\ 7 Division of Endocrinology, Department of Internal Medicine, Shandong Provincial Qianfoshan Hospital, Ji'nan, China \\ Corresponding Author: Cuiyan Wang \\ Email address: 13869181997@163.com
}

Background. Mapping techniques using cardiac magnetic resonance imaging have significantly improved the diagnostic accuracy for myocarditis with focal myocardial injuries. The aim of our study was to determine whether T1 and T2 mapping techniques could identify diffuse myocardial injuries in "normal-appearing" myocardium in pediatric patients with clinically suspected myocarditis and to evaluate the associations between diffuse myocardial injuries and cardiac function parameters.

Methods. Forty-six subjects were included in this study: 20 acute myocarditis patients, 11 subacute/chronic myocarditis patients and 15 control children. T2 values, native T1 values and the extracellular volume (ECV) of "normal-appearing" myocardium were compared among the three groups of patients. Associations between diffuse myocardial injuries and cardiac function parameters were also evaluated.

Results. The ECV of "normal-appearing" myocardium was significantly higher in the subacute/chronic myocarditis group than in the control group ( $30.1 \pm 0.9$ vs $27.0 \pm 0.6, P=0.004)$. No significant differences in T1 and T2 values between the acute myocarditis and control groups were found. In the subacute/chronic myocarditis group, a significant association between ECV and left ventricle ejection fraction was found $(P=0.03)$.

Conclusions. Diffuse myocardial injuries are likely to occur in subacute/chronic myocarditis patients with prolonged inflammatory responses. Mapping techniques have great value for the diagnosis and monitoring of myocarditis. 
1 Identifying myocardial injuries in "normal-appearing" myocardium in

2 pediatric patients with clinically suspected myocarditis using mapping

3 techniques

4

5 Haipeng Wang ${ }^{1}$, Bin Zhao ${ }^{2}$, Huan Yang ${ }^{1}$, Tianyi Qian³, Bo Han4, Haipeng

$6 \mathrm{Jia}^{5}$, Jing $\mathrm{An}^{6}$, Junyu Zhao ${ }^{7}$, Ximing Wang ${ }^{1}$, Cuiyan Wang ${ }^{2}$ *

71 Department of Radiology, Shandong Provincial Hospital affiliated to

8 Shandong First Medical University, Jinan, China

92 Shandong Medical Imaging Research Institute Affiliated to Shandong

10 University, Ji'nan, China

$11{ }^{3}$ Siemens Healthcare, MR Collaborations NE Asia, Beijing, China

124 Department of Pediatrics, Shandong Provincial Hospital affiliated to

13 Shandong First Medical University, Jinan, China

145 Department of Radiology, Qilu Hospital of Shandong University, Jinan,

15 China

$16{ }^{6}$ Siemens SSMR, APPL, Beijing, China

7 Division of Endocrinology, Department of Internal Medicine, Shandong Provincial Qianfoshan Hospital, Jinan, China

${ }^{*}$ Corresponding Author: 
21 Cuiyan Wang

22 No. 324, Jingqi Road, Huaiyin District, Ji'nan City, Shandong Province,

23250000 , China

24 E-mail address: 13869181997@163.com 


\section{Abstract}

Background. Mapping techniques using cardiac magnetic resonance imaging have significantly improved the diagnostic accuracy for myocarditis with focal myocardial injuries. The aim of our study was to determine whether T1 and T2 mapping techniques could identify diffuse myocardial injuries in "normal-appearing" myocardium in pediatric patients with clinically suspected myocarditis and to evaluate the associations between diffuse myocardial injuries and cardiac function parameters.

Methods. Forty-six subjects were included in this study: 20 acute myocarditis patients, 11 subacute/chronic myocarditis patients and 15 control children. T2 values, native T1 values and the extracellular volume (ECV) of "normal-appearing" myocardium were compared among the three groups of patients. Associations between diffuse myocardial injuries and cardiac function parameters were also evaluated.

Results. The ECV of "normal-appearing" myocardium was significantly higher in the subacute/chronic myocarditis group than in the control group (30.1 \pm 0.9 vs $27.0 \pm 0.6, P=0.004$ ). No significant differences in T1 and T2 values between the acute myocarditis and control groups were found. In the subacute/chronic myocarditis group, a significant association between ECV and left ventricle ejection fraction was found $(P=0.03)$. 
46 Conclusions. Diffuse myocardial injuries are likely to occur in

47 subacute/chronic myocarditis patients with prolonged inflammatory

48 responses. Mapping techniques have great value for the diagnosis and

49 monitoring of myocarditis. 
50 Introduction

51 Myocarditis is a myocardial inflammatory disease associated with various

52 injuries, clinical manifestations and outcomes. ${ }^{[1,2,3]}$ Myocarditis has been

53 identified as a significant cause of sudden death in children. [4, 5] In addition,

54 myocarditis may be an underlying cause of dilated cardiomyopathy (DCM)

55 (up to $40 \%$ of DCM cases are caused by myocarditis) ${ }^{[6]}$ and may result in

56 death or cardiac transplantation as long as 12 years after diagnosis. ${ }^{[7]}$

57 Cardiac magnetic resonance (CMR) has been an established noninvasive

58 tool for the diagnosis and evaluation of myocarditis. ${ }^{8]}$ Conventional CMR

59 imaging, including T2 weighted imaging, T1 weighted imaging and late

60 gadolinium enhancement (LGE) imaging, is most useful for evaluating focal

61 myocardial injuries by visually comparing the affected area with the normal

62 myocardium. ${ }^{[9-10]}$ These combined imaging sequences are an essential part

63 of the "Lake Louise" criteria (2009) [9] and have considerable diagnostic

64 accuracy in myocarditis patients with angina-like symptoms and recent

65 symptom onset [11-13]. Diffuse myocardial injuries in myocarditis may

66 present as "normal-appearing" myocardium if it is not compared to normal

67 myocardium. It can also be quantitatively analyzed by normalizing the signals of the myocardium to remote myocardium or skeletal muscles, although the diagnostic accuracy might be affected by abnormal signals of 
70 reference muscles ${ }^{[9,14]}$.

71 Currently, T1 and T2 mapping techniques are applied to determine the 72 diagnosis and prognosis of myocarditis. Mapping techniques offer a 73 quantitative assessment of the myocardium by using standardized, reproducible T1 and T2 values and have the potential to identify both focal and diffuse myocardial injuries from myocarditis [10]. Extracellular volume (ECV), which is derived from the ratio of pre- and postcontrast T1 values, can measure the fraction of volume occupied by the extracellular space in the myocardium and has become a marker of myocardial tissue remodeling [15]. In 2018, updated "Lake Louise" criteria [16] were published, and parametric mapping techniques were included in the diagnostic criteria for myocardial inflammation. Compared with that of the original "Lake Louise" criteria, significantly improved diagnostic accuracy has been reported in patients with myocarditis using mapping techniques $[12-13,17]$. In addition, diffuse myocardial injuries in DCM ${ }^{[18]}$, myocardial infarctions ${ }^{[19-20]}$ and heart failure ${ }^{[21-22]}$ have been reported by mapping techniques. Myocarditis with focal myocardial injuries has been fully shown using conventional CMR and mapping techniques. However, myocarditis with "normal-appearing" myocardium that might result from diffuse myocardial injuries has received less attention. 
90 In our study, we focused on the "normal-appearing" myocardium-

91 myocardium without focal myocardial edema or necrosis/fibrosis visible on

92 conventional CMR-in pediatric patients with clinically suspected

93

myocarditis. We attempted to determine whether T1 and T2 mapping techniques could identify diffuse myocardial injuries in pediatric myocarditis patients, and we then evaluated whether there were associations between diffuse myocardial injuries and cardiac function in these patients.

\section{Materials and methods}

In this prospective study, pediatric patients with clinically suspected myocarditis from Feb 2016 to Jan 2018 in our hospital were included. All patients were diagnosed by an experienced pediatrician according to the myocardial diagnostic criteria proposed by the European Society of Cardiology Working Group on Myocardial and Pericardial Diseases [23]. According to the duration of symptoms from onset to CMR examinations, myocarditis patients were divided into 2 groups: the acute myocarditis (AM) group ( $\leq 3$ months) and the subacute/chronic myocarditis $(\mathrm{CM})$ group ( $>3$ months). Exclusion criteria were contraindications for CMR, coronary artery diseases, congenital heart diseases, cardiomyopathies, or other medical history of cardiac disease [11]. Clinical manifestations, immunological 
110 features and electrocardiography (ECG) results were recorded. Children

111 with some mild nonspecific symptoms (such as fatigue, chest congestion)

112 performed CMR examinations to rule out myocarditis. We included 15

113 control children with normal immunological features, electrocardiography

114 (ECG) and CMR in our study as normal group (NC group). The study was

115 approved by the ethics committee of Shandong Medical Imaging Research

116 Institute, and written informed consent was obtained from the parents of

117 pediatric patients (No. 2016-001).

118

\section{CMR imaging protocol}

CMR imaging was performed using a MAGNETOM Skyra 3T MR scanner

(Siemens Healthcare, Erlangen, Germany) with an 18-channel body matrix coil. All data acquired was retrospectively gated based on the ECG results. Respiratory gating was applied in pediatric patients who could cooperate for a breath-hold during the CMR examinations (usually patients older than 6 years old), and CMR images were acquired at the end-expiratory point. Patients who could not hold their breath (usually younger than 6 years old) were sedated with $10 \%$ chloral hydrate solution and examined under freebreathing conditions.

The CMR imaging protocols included T2-weighted imaging, LGE imaging, 
130 cine imaging and quantitative image mapping. T2-weighted turbo inversion 131 recovery magnitude (TIRM) sequences were performed in the short-axis 132 (SA) and horizontal long-axis (HLA) orientations (repetition time (TR)/echo 133 time $(T E)=800 / 44 \mathrm{~ms}$, flip angle $(F A)=180^{\circ}$, field of view $(F O V)=300 \times 225$ $134 \mathrm{~mm}^{2}$, and voxel size $\left.=1.3 \times 1.3 \times 6 \mathrm{~mm}^{3}\right)$. LGE imaging was performed using 135 phase-sensitive inversion recovery (PSIR) sequences in the HLA and SA 136 orientations $7-10$ minutes after intravenous administration of $0.2 \mathrm{mmol} / \mathrm{kg}$ 137 Gd-DTPA (Magnevist, Bayer, Germany). The parameters were as follows: $138 \mathrm{TR} / \mathrm{TE}=448 / 2.0 \mathrm{~ms}, \mathrm{FOV}=300 \times 350 \mathrm{~mm}^{2}$, matrix $=256 \times 192$, and voxel 139 size $=1.4 \times 1.4 \times 6 \mathrm{~mm}^{3}$. The inversion time $(\mathrm{TI})$ of LGE imaging was 140 determined by using the TI scout. Steady-state free precession (SSFP) cine 141 images were acquired in the HLA and sequential SA orientations from 142 ventricular base to apex with the following imaging parameters: $143 \mathrm{TR} / \mathrm{TE}=39.2 / 1.4, \mathrm{FA}=80^{\circ}, \mathrm{FOV}=300 \times 225 \mathrm{~mm}^{2}$, and voxel size $=1.6 \times 1.6 \times 6.0$ $\left.144 \mathrm{~mm}^{3}\right)$.

145 T1 mapping was performed with 3(3)5 modified Look-Locker inversion 146 recovery (MOLLI) sequences in the HLA and three SA orientations (basal, 147 mid and apical ventricular SA planes) ${ }^{[12,24]}$ before and 15 minutes after $\mathrm{Gd}$ 148 DTPA administration with the following parameters: $T R / T E=2.4 / 1.1 \mathrm{~ms}$, $149 \mathrm{FA}=35^{\circ}, \mathrm{FOV}=300 \times 225 \mathrm{~mm}^{2}$, acquisition matrix $=256 \times 192 \mathrm{~mm}^{2}$, and voxel 
150 size $=1.4 \times 1.4 \times 8.0 \mathrm{~mm}^{3}$. T2 mapping was acquired using a SSFP sequence

151 with three different T2 preparation times in the HLA and three SA 152 orientations (basal, mid and apical ventricular SA planes). The parameters 153 were as follows: TE=0 ms, $25 \mathrm{~ms}, 55 \mathrm{~ms}$; TR=3 $\mathrm{TRR}$; $\mathrm{FA}=50^{\circ}$; $\mathrm{FOV}=$ $154300 \times 225 \mathrm{~mm}^{2}$; acquisition matrix $=256 \times 384 \mathrm{~mm}^{2}$; and voxel size= $155 \quad 0.9 \times 0.9 \times 8.0 \mathrm{~mm}^{3}$.

\section{CMR image analysis}

All the original image data were processed on the workstation (Siemens Medical Systems). Two experienced CMR radiologists (C.Y.W and H.P.W), who were blinded to patient information, independently analyzed all CMR images.

Left ventricular (LV) cardiac function parameters were evaluated in the 163 cine images. LV endocardial and epicardial contours were drawn manually for each diastolic and systolic frame in the sequential SA cine images, and LV cardiac function parameters, including end-diastolic volume (EDV), endsystolic volume (ESV), left ventricle ejection fraction (LVEF), LV mass and stroke volume (SV), were automatically acquired on the workstation. LV cardiac function parameters were standardized as follows [25]:

Standardized LV cardiac function parameters $=$ LV cardiac function 
171 The papillary muscles and trabeculations were included as part of 172 ventricular cavity ${ }^{[26] .}$

173 Myocardial edema and necrosis/fibrosis were defined by visual 174 assessment in the T2-weighted images and LGE images. The presence and 175 location of myocardial edema or fibrosis were independently evaluated by 176 two CMR radiologists according to the 17-segment model proposed by the 177 American Heart Association (AHA) ${ }^{[27]}$. For the contradictory findings 178 regarding myocardial injuries after independent evaluation, two CMR 179 radiologists would discuss the findings together and reach a consensus. 180 The myocardium without edema or necrosis/fibrosis was defined as 181 "normal-appearing" myocardium, which might include normal myocardium 182 and abnormal myocardium with diffuse myocardial injuries.

183 T1 and T2 values of "normal-appearing" myocardium in the HLA and SA orientations were measured directly in their T1 and T2 maps. Endocardial 185 and epicardial borders were carefully contoured to exclude artifacts, 186 epicardial fat and blood pools. Then, the T2 values, native T1 values, and postcontrast T1 values of "normal-appearing" myocardium in the HLA and 188 three SA orientations were acquired (Fig 1). T1 and T2 values in three SA 189 orientations were averaged for data analysis. The extracellular volume 
190 (ECV) of the "normal-appearing" myocardium was calculated using native 191 and postcontrast T1 values of the myocardium and blood pools as well as 192 hematocrit (HCT), as follows ${ }^{[8]}$ :

$$
\text { ECV }(\%)=(1-H C T) \times(\Delta R 1 \text { of myocardium } / \Delta R 1 \text { of blood pool })
$$

195 The native and postcontrast T1 values of the blood pools were also 196 measured directly in the LV cavity, avoiding the papillary muscle. 197 Myocardium with higher T1 and T2 values and ECV was identified as abnormal myocardium with diffuse myocardial injuries.

\section{Statistical analysis}

All analyses were performed using the Statistical Package for the Social

Sciences (SPSS, version

and

Empower Stats

203 (http://www.empowerstats.com) software. Categorical data were reported as percentages (\%), and continuous data were reported as the mean \pm standard deviation (SD) or median (range). The Shapiro-Wilk test was used to assess the normality of the variables using SPSS. Clinical characteristics in the $\mathrm{AM}, \mathrm{CM}$ and $\mathrm{NC}$ groups were compared using chi-square tests for categorical variables, one-way analysis of variance (ANOVA) for normally distributed continuous variables, and the Kruskal-Wallis test for nonnormally 
210 distributed continuous variables. The interaction test and covariate

211 screening were performed to adjust for patient-specific factors using

212 Empower Stats. Linear regression analyses were used to compare T2

213 values, native T1 values and the ECV of "normal-appearing" myocardium in

214 the HLA and SA orientations among the AM, CM and NC groups. The

215 influence of focal myocardial injuries on T1 and T2 values and the ECV of

216 "normal-appearing" myocardium in myocarditis patients were also evaluated

217 using linear regression analyses. Associations between ECV and LV

218 cardiac function parameters in the AM and CM groups were evaluated using

219 multiple linear regression analyses with adjustment for confounding 220 variables, and odds ratios (ORs) and 95\% confidence intervals (Cls) were 221 calculated.

222 All statistical tests were two-sided, and $P$-values less than 0.05 were 223 considered statistically significant.

Results

226 Twenty pediatric patients with acute myocarditis (10 male; median age, 9

227 years old), 11 patients with subacute/chronic myocarditis (9 male; median 228 age, 6 years old) and 15 control children ( 9 male; median age, 11 years old) 229 were included in the study. 


\section{Clinical characteristics}

232 The clinical characteristics of all pediatric patients are shown in Table 1.

233 The most common clinical manifestation in the AM group was chest 234 pain/distress, which was present in eight AM patients (40.0\%). In the CM 235 group, six patients $(54.5 \%)$ experienced palpitation. Abnormal cardiac 236 troponin $\mathrm{T}(\mathrm{cTnT})$ or brain natriuretic peptide (BNP) levels were observed in 237 nine $\mathrm{AM}$ patients (45.0\%) and seven $\mathrm{CM}$ patients (63.6\%). ECG 238 abnormalities were detected in all AM patients and ten CM patients (90.9\%). 239 The most common ECG finding in the AM group was ST-T changes (45.0\%), 240 while ventricular premature beats (VPBs) were common in the CM group $241(45.5 \%)$.

CMR findings

The standard LV cardiac function parameters and myocardial tissue 245 characterizations in the AM, CM and NC groups are shown in Table 2. Two 246 AM patients and two CM patients were sedated and underwent CMR 247 examinations under free-breathing conditions, and the remaining patients 248 underwent CMR examinations with respiratory gating. There were no statistically significant differences in standardized EDV, ESV, LV mass, SV 
250 and LVEF among the AM, CM and NC groups. Regional myocardial edema 251 or necrosis/fibrosis was found in $7 \mathrm{AM}$ patients $(35.0 \%)$ and four $\mathrm{CM}$ 252 patients $(36.4 \%)$.

253 The $\mathrm{T} 1$ and $\mathrm{T} 2$ values and ECV of "normal-appearing" myocardium in the $254 \mathrm{AM}, \mathrm{CM}$ and NC groups are shown in Table 2 and Fig 2. Compared with 255 that of the NC group, the ECV of the "normal-appearing" myocardium was 256 significantly higher in the CM group after adjusting for BSA, sex, heart rate 257 or HCT (SA: $30.1 \pm 0.9$ vs $27.0 \pm 0.6, P=0.004$ ). No significant differences in $258 \mathrm{~T} 1$ and T2 values were found between the AM and NC groups.

259 To study whether focal myocardial injuries would influence the T1 and T2 260 values and ECV of "normal-appearing" myocardium, we divided myocarditis 261 patients into another two groups: myocarditis patients with focal myocardial 262 injuries (35.5\%) and without focal myocardial injuries (64.5\%). No significant 263 differences in T1 or T2 values or ECV between the two groups were found. 264 The T1 and T2 values and ECV of "normal-appearing" myocardium in 265 myocarditis patients with and without focal myocardial injuries are shown in 266 Table 3.

267 In the CM group, we found negative associations between ECV and $268 \operatorname{LVEF}(\mathrm{OR},-0.4 ; 95 \% \mathrm{Cl},-0.7,-0.1 ; P=0.03)$ after adjusting for age, sex, 269 heart rate or HCT. The associations between ECV and LV cardiac function 
270 parameters in the CM group are shown in Table 4.

271

\section{Discussion}

273 In our study, we evaluated the tissue-related changes of "normal274 appearing" myocardium in pediatric patients with clinically suspected 275 myocarditis using mapping techniques. We found that ECV could detect 276 diffuse myocardial injuries in "normal-appearing" myocardium in pediatric CM patients, and ECV was associated with LVEF. Therefore, mapping techniques could increase the sensitivity of CMR for monitoring diffuse myocardial injuries in patients with clinically suspected myocarditis.

Mapping technologies could be influenced by numerous factors, including 281 the MR scanner, magnetic field strength, exact sequence used, image 282 acquisition plane, contrast agent dose and patient's physiological 283 differences [10, 19, 28-30]. In our study, the scanning protocols of the different mapping technologies were identical, and interaction tests and covariate screenings were performed to adjust for BSA, sex, age, heart rate and HCT of subjects and could minimize the influence of confounding variables.

287 LGE has been an established noninvasive tool to evaluate focal 288 myocardial necrosis/fibrosis and has shown excellent correlation with 289 pathology ${ }^{[8]}$. In acute "infarct-like" myocarditis, a high sensitivity of LGE has 
290 been reported [31]. However, it is not very sensitive in very mild myocarditis

291 cases, which might have diffuse myocardial tissue-related changes. In

292 contrast to LGE, ECV is well suited to measure focal and diffuse myocardial

fibrosis and exhibits the best agreement with histological measures of the

294

295

296

297

298

299

300

301

302

303

304

305

306

307

308

309

collagen volume fraction. ECV has been shown to be reproducible, predict outcomes and provide "added prognostic value" in myocardial disease ${ }^{[16,32]}$. For myocarditis, ECV has been included in the updated "Lake Louise" criteria ${ }^{[16]}$, which could certainly greatly improve the diagnostic sensitivity for myocarditis over that of the original "Lake Louise" criteria, especially in myocarditis with diffuse myocardial injuries.

In our study, we found that the ECV of "normal-appearing" myocardium was significantly higher in pediatric CM patients than in NC patients, which indicated diffuse myocardial injuries. Myocarditis has been identified as an underlying cause of DCM, and up to $40 \%$ of DCM cases are caused by myocarditis [6]. The availability of murine models of myocarditis has facilitated much of our understanding of the pathogenesis of myocarditisDCM [33]. During the progression of myocarditis, inflammatory cells embedded in the interstitial matrix contribute to the inflammatory response and cardiac remodeling. The expansive interstitial matrix could be measured by ECV [15]. In our study, diffuse myocardial injuries were more 
310 likely to occur in CM patients than in AM patients. Abnormal cTnT or BNP

311 was observed in $63.6 \%$ of CM patients during CMR examination, which was

312 higher than that in AM patients (45.0\%). We hypothesized that expansive 313 interstitial matrix deposition was likely to occur in $\mathrm{CM}$ patients with 314 prolonged and recurrent inflammatory responses. ECV might be a marker of 315 myocarditis leading to DCM. The values of ECV related to the outcomes of 316 CM patients have been followed up and will be discussed in future studies. 317 ECV quantification of interstitial expansion remains a powerful tool to 318 investigate diffuse myocardial injuries.

319 In our study, native T1 values of the "normal-appearing" myocardium 320 were not significantly higher in $\mathrm{AM}$ and $\mathrm{CM}$ patients than in NC patients, which was inconsistent with recent data by Radunski UK et al. ${ }^{[14]}$. Radunski UK et al. found that native T1 values in the "normal-appearing" myocardium of AM patients were significantly higher than the reference values from the 324 myocardium of healthy volunteers. This discrepancy could be explained by the fact that all the AM patients who Radunski UK included had typical focal myocardial LGE findings, while the inflammatory response in our study was

327 mild. Despite this, a higher ECV of "normal-appearing" myocardium was 328 observed in the CM group. Native T1 values perform as composite 329 indicators of both intracellular and extracellular compartments [34] and, 
330 therefore, can be less sensitive to increased extracellular space or more

331 sensitive to other characteristics of the tissue (such as increased iron

332 content, fatty deposition, and edema) ${ }^{[34]}$. ECV is derived from the ratio of T1

333 signal values and simply quantifies the interstitial presence of gadolinium 334 relative to plasma [15]. ECV represents a physiological parameter, and its 335 values are therefore reproducible. Therefore, ECV could reflect diffuse 336 myocardial injuries with more sensitivity than native T1 values.

337 In our study, T2 values in the "normal-appearing" myocardium of AM 338 patients was not significantly higher than those in the NC group, which was 339 in agreement with the findings of Radunski UK et al. [14]. In addition to the 340 disadvantages of T2 mapping due to unstable myocardial edema ${ }^{[14]}$, we 341 also reasoned that mild myocardial inflammation in our study would have 342 influenced the results.

343 In our study, we found associations between ECV and LVEF in CM 344 patients, which have also been reported in patients with diabetic 345 cardiomyopathy, myocardial infarction, hypertrophic cardiomyopathy and 346 heart failure ${ }^{[18-22]}$. The pathophysiology of myocarditis in murine models 347 suggests that a persistent inflammatory response in the chronic phase of 348 myocarditis leads to ventricular remodeling, which is characterized by 349 myocyte hypertrophy, myocyte apoptosis, contractile dysfunction and 
350 extracellular matrix volume expansion $[1,2,17,35]$. ECV has become a marker

351 of myocardial tissue remodeling [15], and it could predict outcomes and 352 provide "added prognostic value" in myocardial disease [16, 32]. Early data 353 indicate that ECV appears to be as prognostically important as LVEF [36, 37], 354 which underestimates the biological importance of the interstitium. In our 355 study, CM patients had increased ECV and normal LVEF. ECV is 356 comparable to LVEF as a marker to evaluate myocardial injuries.

357 This study has several potential limitations. First, the myocarditis patients 358 involved in our study were diagnosed according to the diagnostic criteria for clinically suspected myocarditis proposed by the ESC Working Group on Myocardial and Pericardial Diseases ${ }^{[23]}$. Endomyocardial biopsy should be the gold standard for the definitive diagnosis of myocarditis. However, it 362 might be unrealistic to perform biopsies in most pediatric myocarditis patients. In our study, $85.0 \%$ AM patients and $72.7 \% \mathrm{CM}$ patients fulfilled 364 more than 3 criteria for clinically suspected myocarditis, which increased the 365 strength of the suspicion for myocarditis. Second, the severity of myocardial 366 inflammation was less severe than that reported in other studies. We did not acquire significant results with native T1 mapping and T2 mapping. The 368 diagnostic efficacy for myocarditis according to the "Lake Louise" criteria was low. Third, the intervals from onset to CMR examinations varied based 
370 on the patient's condition, which might have influenced the CMR findings.

371 Fourth, the number of pediatric myocarditis patients included in the study

372 was limited. Analyses of larger populations should be performed in the 373 future.

\section{Conclusion}

In conclusion, diffuse myocardial injuries are likely to occur in CM patients with prolonged inflammatory responses. Mapping techniques have great value for the diagnosis and monitoring of myocarditis.

\section{Acknowledgements}

We would like to acknowledge the participation of the study patients and their families. We also wish to acknowledge the support of department of pediatrics. 
1. Kindermann I, Barth C, Mahfoud F, Ukena C, Lenski M, Yilmaz A, Klingel

2. Cooper LT Jr. Myocarditis. N Engl J Med. 2009; 360:1526 -1538.

3. Zagrosek A, Abdel-Aty $H$, Boyé $P$, Wassmuth R, Messroghli D, Utz W,

6. Heymans S, Eriksson U, Lehtonen J, Cooper LT Jr. The Quest for New Approaches in Myocarditis and Inflammatory Cardiomyopathy. J Am Coll Cardiol. 2016; 68:2348-2364.

7. Towbin JA, Lowe AM, Colan SD, Sleeper LA, Orav EJ, Clunie S, Messere J, Cox GF, Lurie PR, Hsu D, Canter C, Wilkinson JD, Lipshultz SE. Incidence, causes, and outcomes of dilated cardiomyopathy in children. JAMA. 2006; 296: 1867-1876. 
405 8. Luetkens JA, Doerner J, Thomas DK, Dabir D, Gieseke J, Sprinkart AM, 406 Fimmers R, Stehning C, Homsi R, Schwab JO, Schild H, Naehle CP. 407 408

9. Friedrich MG, Sechtem U, Schulz-Menger J, Holmvang G, Alakija P, Cooper LT, White JA, Abdel-Aty H, Gutberlet M, Prasad S, Aletras A, Laissy JP, Paterson I, Filipchuk NG, Kumar A, Pauschinger M, Liu P; International Consensus Group on Cardiovascular Magnetic Resonance in Myocarditis. Cardiovascular magnetic resonance in myocarditis: A JACC White Paper. J Am Coll Cardiol. 2009; 53:1475-1487.

10. Hamlin SA, Henry TS, Little BP, Lerakis S, Stillman AE. Mapping the future of cardiac MR imaging: case-based review of T1 and T2 mapping techniques. Radiographics. 2014; 34:1594-1611.

11.Luetkens JA, Homsi R, Dabir D, Kuetting DL, Marx C, Doerner J, Schlesinger-Irsch U, Andrié R, Sprinkart AM, Schmeel FC, Stehning C, Fimmers R, Gieseke J, Naehle CP, Schild HH, Thomas DK. Comprehensive Cardiac Magnetic Resonance for Short-Term Follow-Up in Acute Myocarditis. J Am Heart Assoc. 2016; 5.

12. Lurz P, Luecke C, Eitel I, Föhrenbach F, Frank C, Grothoff M, de Waha S, Rommel KP, Lurz JA, Klingel K, Kandolf R, Schuler G, Thiele H, 
425

426

427

428

429

430

431

432

433

434

435

436

437

438

439

440

441

442

443

444

Gutberlet M. Comprehensive Cardiac Magnetic Resonance Imaging in Patients With Suspected Myocarditis: The MyoRacer-Trial. J Am Coll Cardiol. 2016; 67:1800-1811.

13. Radunski UK, Lund GK, Stehning C, Schnackenburg B, Bohnen S, Adam G, Blankenberg S, Muellerleile K. CMR in patients with severe myocarditis: diagnostic value of quantitative tissue markers including extracellular volume imaging. JACC Cardiovasc Imaging. 2014; 7:667675.

14.Radunski UK, Lund GK, Säring D, Bohnen S, Stehning C, Schnackenburg B, Avanesov M, Tahir E, Adam G, Blankenberg S, Muellerleile K. T1 and T2 mapping cardiovascular magnetic resonance imaging techniques reveal unapparent myocardial injury in patients with myocarditis. Clin Res Cardiol. 2017; 106:10-17.

15. Haaf P, Garg P, Messroghli DR, Broadbent DA, Greenwood JP, Plein S. Cardiac T1 Mapping and Extracellular Volume (ECV) in clinical practice: a comprehensive review. J Cardiovasc Magn Reson. 2016; 18:89.

16. Ferreira VM, Schulz-Menger J, Holmvang G, Kramer CM, Carbone I, Sechtem U, Kindermann I, Gutberlet M, Cooper LT, Liu P, Friedrich MG. Cardiovascular Magnetic Resonance in Nonischemic Myocardial Inflammation: Expert Recommendations. J Am Coll Cardiol. 2018; 
445

446

447

448

449

450

451

452

453

454

455

456

457

458

459

460

461

462

463

464

72:3158-3176.

17. Hinojar R, Foote L, Arroyo Ucar E, Jackson T, Jabbour A, Yu CY, McCrohon J, Higgins DM, Carr-White G, Mayr M, Nagel E, Puntmann VO. Native T1 in discrimination of acute and convalescent stages in patients with clinical diagnosis of myocarditis: a proposed diagnostic algorithm using CMR. JACC Cardiovasc Imaging. 2015; 8:37-46.

18. Hong YJ, Park CH, Kim YJ, Hur J, Lee HJ, Hong SR, Suh YJ, Greiser A, Paek MY, Choi BW, Kim TH. Extracellular volume fraction in dilated cardiomyopathy patients without obvious late gadolinium enhancement: comparison with healthy control subjects. Int J Cardiovasc Imaging. 2015; $31: 115-122$.

19. Ugander M, Oki AJ, Hsu LY, Kellman P, Greiser A, Aletras AH, Sibley CT, Chen MY, Bandettini WP, Arai AE. Extracellular volume imaging by magnetic resonance imaging provides insights into overt and sub-clinical myocardial pathology. Eur Heart J. 2012; 33:1268-1278.

20. Chan W, Duffy SJ, White DA, Gao XM, Du XJ, Ellims AH, Dart AM, Taylor AJ. Acute left ventricular remodeling following myocardial infarction: coupling of regional healing with remote extracellular matrix expansion. JACC Cardiovasc Imaging. 2012; 5:884-893.

21. Mascherbauer J, Marzluf BA, Tufaro C, Pfaffenberger S, Graf A, 
465

466

467

468

Wexberg P, Panzenböck A, Jakowitsch J, Bangert C, Laimer D, Schreiber C, Karakus G, Hülsmann M, Pacher R, Lang IM, Maurer G, Bonderman D. Cardiac magnetic resonance postcontrast T1 time is associated with outcome in patients with heart failure and preserved ejection fraction. Circ Cardiovasc Imaging. 2013; 6:1056-1065.

22. Iles LM, Ellims AH, Llewellyn H, Hare JL, Kaye DM, McLean CA, Taylor AJ. Histological validation of cardiac magnetic resonance analysis of regional and diffuse interstitial myocardial fibrosis. Eur Heart $\mathrm{J}$ Cardiovasc Imaging. 2015; 16:14-22.

23. Caforio AL, Pankuweit S, Arbustini E, Basso C, Gimeno-Blanes J, Felix SB, Fu M, Heliö T, Heymans S, Jahns R, Klingel K, Linhart A, Maisch B, McKenna W, Mogensen J, Pinto YM, Ristic A, Schultheiss HP, Seggewiss H, Tavazzi L, Thiene G, Yilmaz A, Charron P, Elliott PM; European Society of Cardiology Working Group on Myocardial and Pericardial Diseases. Current state of knowledge on aetiology, diagnosis, management, and therapy of myocarditis: a position statement of the European Society of Cardiology Working Group on Myocardial and Pericardial Diseases. Eur Heart J. 2013; 34:2636-2648.

24. Hwang SH, Choi EY, Park CH, Paek MY, Greiser A, Kim TH, Choi BW. Evaluation of extracellular volume fraction thresholds corresponding to 
485

486

487

488

489

490

491

492

493

494

495

496

497

498

499

500

501

502

503

504

myocardial late-gadolinium enhancement using cardiac magnetic resonance. Int J Cardiovasc Imaging. 2014; 30:137-144.

25. Kawel-Boehm N, Maceira A, Valsangiacomo-Buechel ER, VogelClaussen J, Turkbey EB, Williams R, Plein S, Tee M, Eng J, Bluemke DA. Normal values for cardiovascular magnetic resonance in adults and children. J Cardiovasc Magn Reson. 2015; 17:29.

26. Buechel EV, Kaiser T, Jackson C, Schmitz A, Kellenberger CJ. Normal right- and left ventricular volumes and myocardial mass in children measured by steady state free precession cardiovascular magnetic resonance. J Cardiovasc Magn Reson. 2009; 11:19.

27. Cerqueira MD, Weissman NJ, Dilsizian V, Jacobs AK, Kaul S, Laskey WK, Pennell DJ, Rumberger JA, Ryan T, Verani MS; American Heart Association Writing Group on Myocardial Segmentation and Registration for Cardiac Imaging. Standardized myocardial segmentation and nomenclature for tomographic imaging of the heart. A statement for healthcare professionals from the Cardiac Imaging Committee of the Council on Clinical Cardiology of the American Heart Association. Circulation. 2002; 105:539-542.

28. Neilan TG, Coelho-Filho OR, Shah RV, Abbasi SA, Heydari B, Watanabe E, Chen Y, Mandry D, Pierre-Mongeon F, Blankstein R, 
505

506

507

508

509

510

511

512

513

514

515

516

517

518

519

520

521

522

523

524

Kwong RY, Jerosch-Herold M. Myocardial extracellular volume fraction from T1 measurements in healthy volunteers and mice: relationship to aging and cardiac dimensions. JACC Cardiovasc Imaging. 2013; 6:672683.

29. Sado DM, Flett AS, Banypersad SM, White SK, Maestrini V, Quarta G, Lachmann RH, Murphy E, Mehta A, Hughes DA, McKenna WJ, Taylor AM, Hausenloy DJ, Hawkins PN, Elliott PM, Moon JC. Cardiovascular magnetic resonance measurement of myocardial extracellular volume in health and disease. Heart. 2012; 98:1436-1441.

30. Liu CY, Liu YC, Wu C, Armstrong A, Volpe GJ, van der Geest RJ, Liu Y, Hundley WG, Gomes AS, Liu S, Nacif M, Bluemke DA, Lima JAC. Evaluation of age-related interstitial myocardial fibrosis with cardiac magnetic resonance contrast-enhanced T1 mapping: MESA (MultiEthnic Study of Atherosclerosis). J Am Coll Cardiol. 2013; 62:1280-1287. 31. Schwab J, Rogg HJ, Pauschinger M, Fessele K, Bareiter T, Bär I, Loose R. Functional and Morphological Parameters with Tissue Characterization of Cardiovascular Magnetic Imaging in Clinically Verified "Infarct-like Myocarditis". Rofo. 2016; 188:365-373.

32. Messroghli DR, Moon JC, Ferreira VM, Grosse-Wortmann L, He T, Kellman P, Mascherbauer J, Nezafat R, Salerno M, Schelbert EB, Taylor 
525

526

527

528

529

530

531

532

533

534

535

536

537

538

539

540

541

542

543

544

AJ, Thompson R, Ugander M, van Heeswijk RB, Friedrich MG. Clinical recommendations for cardiovascular magnetic resonance mapping of $\mathrm{T} 1$, $\mathrm{T} 2, \mathrm{~T}^{*}$ and extracellular volume: A consensus statement by the Society for Cardiovascular Magnetic Resonance (SCMR) endorsed by the European Association for Cardiovascular Imaging (EACVI). J Cardiovasc Magn Reson. 2017; $19: 75$.

33. Sagar S, Liu PP, Cooper LT Jr. Myocarditis. Lancet 2012; 379:738-47.

34. Burt JR, Zimmerman SL, Kamel IR, Halushka M, Bluemke DA. Myocardial T1 mapping: techniques and potential applications. Radiographics. 2014; 34:377-395.

35. Elamm C 1, Fairweather D, Cooper LT. Pathogenesis and diagnosis of myocarditis. Heart. 2012; 98:835-840.

36. Eitel I, Pöss J, Jobs A, Eitel C, de Waha S, Barkhausen J, Desch S, Thiele H. Left ventricular global function index assessed by cardiovascular magnetic resonance for the prediction of cardiovascular events in ST-elevation myocardial infarction. J Cardiovasc Magn Reson. $2015 ; 17: 62$.

37. Sanguineti F, Garot P, Mana M, O'h-Ici D, Hovasse T, Unterseeh T, Louvard Y, Troussier X, Morice MC, Garot J. Cardiovascular magnetic resonance predictors of clinical outcome in patients with suspected acute 
545 myocarditis. J Cardiovasc Magn Reson. 2015; 17:78.

546 


\section{Figure 1}

Comprehensive cardiac magnetic resonance images of a 14-year-old child with acute myocarditis.

He was hospitalized after four days of chest pain. Regional mid-wall myocardial edema of the interventricular septum and epicardial edema of the anterior, lateral, and inferior walls are shown in the end-diastolic ( $A, I)$ and end-systolic (B, J) cine images and T2-weighted images (C). Regional myocardial necrosis/fibrosis was also found in the identical location on LGE imaging (D, K). In the T2 maps (E, L), native $T 1$ maps $(F, M)$, post-contrast T1 maps $(G, N)$ and ECV maps $(H, O)$, the dotted line shows the ROI of the "normal-appearing" myocardium excluding visible myocardial edema or necrosis/fibrosis on conventional MRI. T1 values, T2 values and ECVs of "normal-appearing" myocardium were measured as follows: T2 values $(H L A)=37.2 \mathrm{~ms}$, Native $T 1$ values $(H L A)=1292.4 \mathrm{~ms}, E C V(H L A)=25.7 \% ; T 2$ values $(S A)=37.8 \mathrm{~ms}$, Native $\mathrm{T} 1$ values $(\mathrm{SA})=1297.5 \mathrm{~ms}$, ECV $(\mathrm{SA})=25.9 \%$.

LGE, late gadolinium enhancement; ECV, extracellular volume; ROI, region of interest; HLA, horizontal long axis; SA, short axis; ECV, extracellular volume.

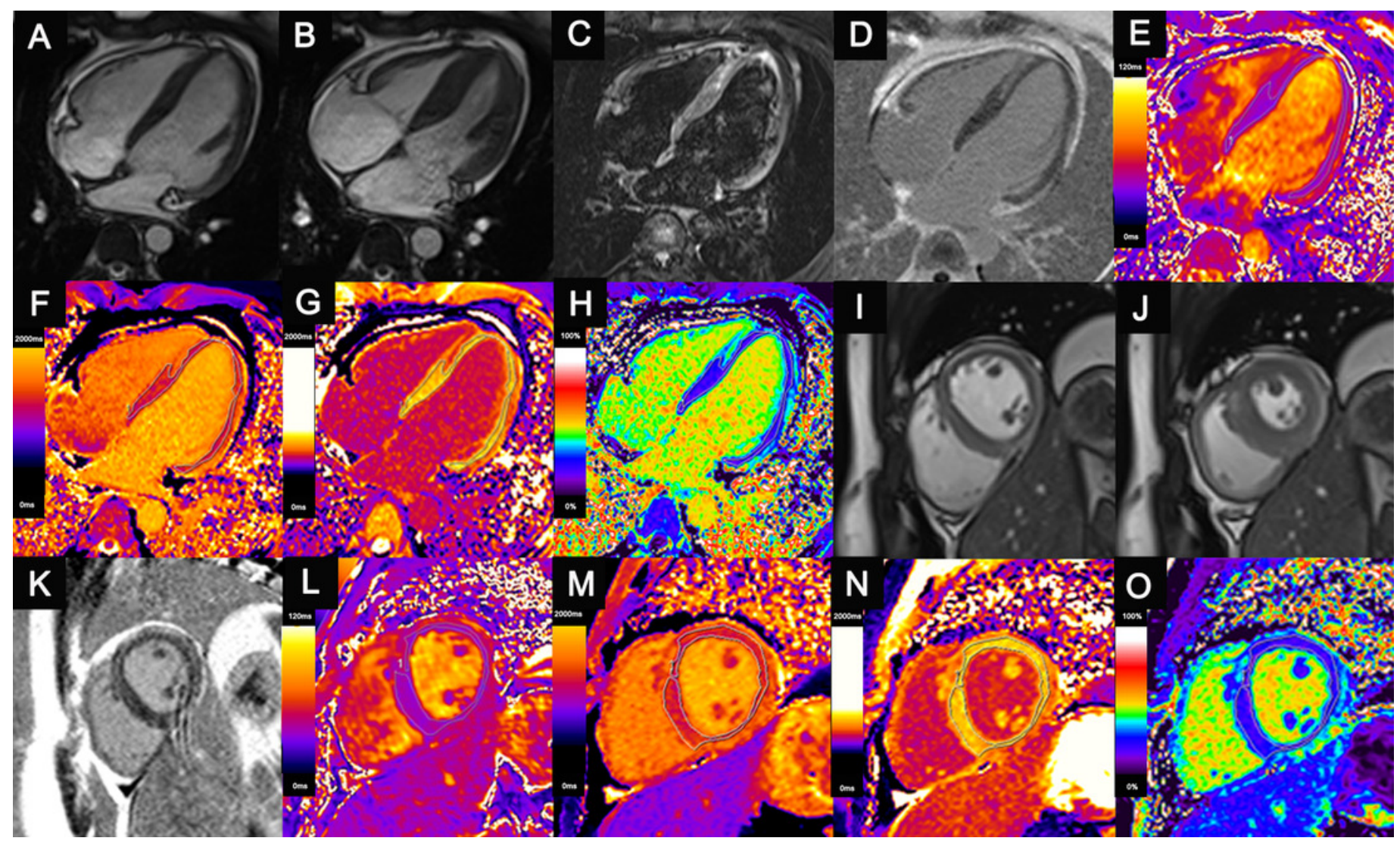


Figure 2

The $\mathrm{T} 1$ and $\mathrm{T} 2$ values of "normal-appearing" myocardium in AM, CM and NC groups.

Compared with NC group, the ECV of "normal-appearing" myocardium significantly increased in CM group (C) after adjusted for BSA, sex, heart rate or HCT (SA: $30.1 \pm 0.9$ VS $27.0 \pm 0.6, P=0.004$ ). No significantly statistical differences were found between $\mathrm{T} 1$ and $\mathrm{T} 2$ values in $\mathrm{AM}$ and $\mathrm{NC}$ group.

AM, acute myocarditis; CM, subacute/chronic myocarditis; NC, normal control; HLA, horizontal long axis; SA, short axis; ECV, extracellular volume

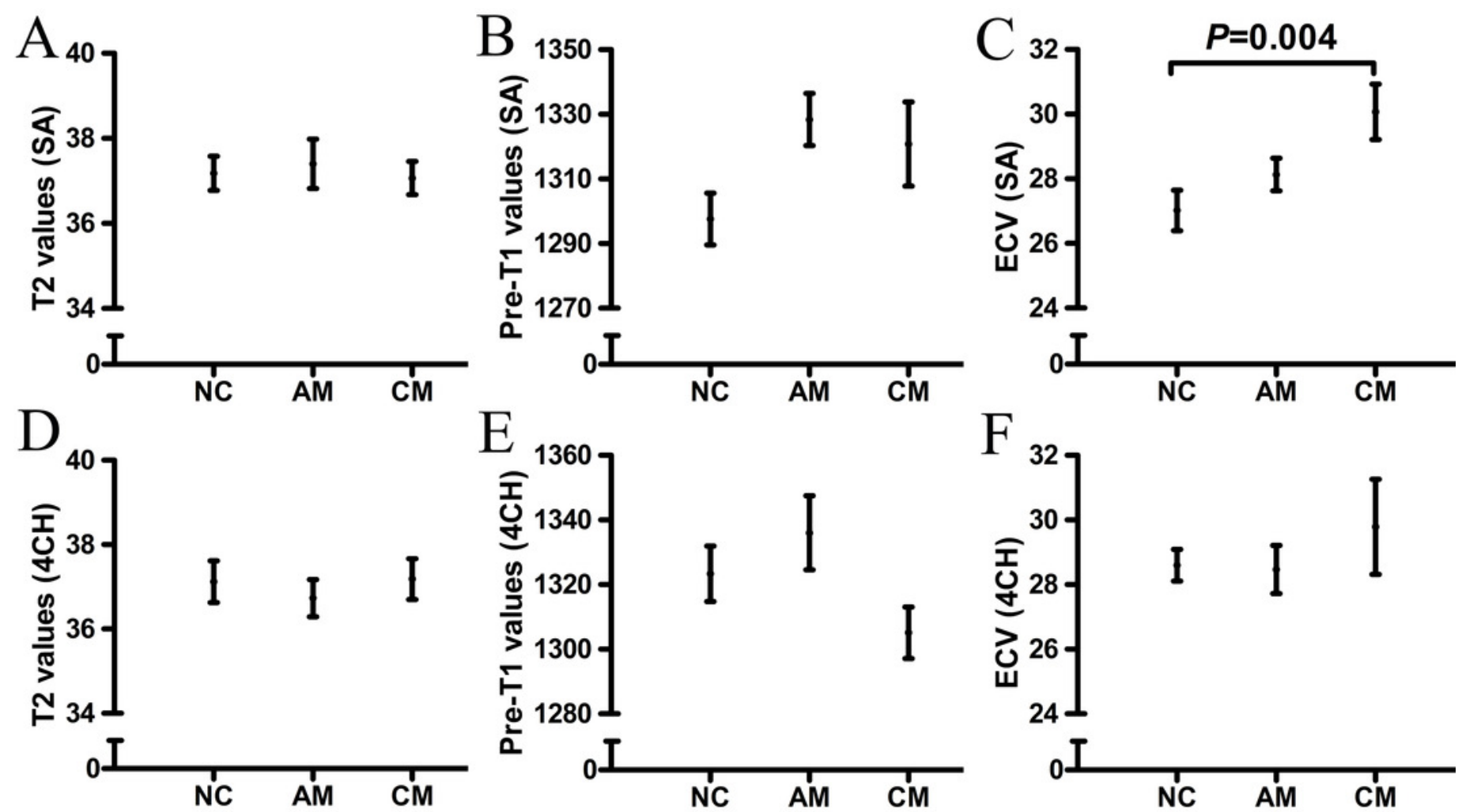




\section{Table $\mathbf{1}$ (on next page)}

\section{Baseline characteristics of children}

Values are presented as $\mathrm{N}(\%)$, mean \pm SE or median (range). *P values $<0.05$

NC, normal control; AM, acute myocarditis; CM, subacute/chronic myocarditis; HCT, hematocrit; HR, Heart rate; CMR, cardiac magnetic resonance; CTnT, cardiac troponin T; BNP, brain natriuretic peptide; ECG, electrocardiography; AVB, atrioventricular block; IVCB, intra-ventricular conduction block; APB, atrial premature beats; VPB, ventricular premature beat. 


\begin{tabular}{|c|c|c|c|c|}
\hline Groups & $\mathrm{NC}(\mathrm{n}=15)$ & $\operatorname{AM}(n=20)$ & $\mathrm{CM}(\mathrm{n}=11)$ & $P$-values \\
\hline $\operatorname{Sex}(M, \%)$ & $9(60.0 \%)$ & $10(50.0 \%)$ & $9(81.8 \%)$ & 0.20 \\
\hline Age $(y)$ & $11(6,13)$ & $9(2,14)$ & $6.0(3,13)$ & $0.02^{*}$ \\
\hline $\mathrm{HCT}(\%)$ & $41.0 \pm 0.5$ & $39.7 \pm 0.8$ & $38.4 \pm 1.0$ & 0.11 \\
\hline $\mathrm{HR}(/ \min )$ & $83.9 \pm 3.9$ & $91.1 \pm 3.5$ & $90.3 \pm 4.6$ & 0.37 \\
\hline Prodrome (\%) & - & $11(55.0 \%)$ & - & - \\
\hline CMR intervals & - & 20 days $(4,60)$ & 8 months $(3,36)$ & - \\
\hline \multicolumn{5}{|c|}{ Clinical manifestations } \\
\hline Fatigue & - & $6(30.0 \%)$ & $4(36.4 \%)$ & 0.72 \\
\hline Palpitation & - & $7(35.0 \%)$ & $6(54.5 \%)$ & 0.29 \\
\hline Chest pain & - & $8(40.0 \%)$ & $4(36.4 \%)$ & 0.84 \\
\hline Dyspnea & - & $3(15.0 \%)$ & $3(27.3 \%)$ & 0.42 \\
\hline Heart failure & - & $2(10.0 \%)$ & 0 & 0.18 \\
\hline \multicolumn{5}{|c|}{ Immunological features } \\
\hline cTNT $(p g / m l)$ & & $3.9(3.0,311.1)$ & $3 \cdot 2(3.0,31.4)$ & 0.33 \\
\hline $\mathrm{BNP}(\mathrm{pg} / \mathrm{ml})$ & & $79.8(5.0,4606.0)$ & $166.1(22.0,488.6)$ & 0.31 \\
\hline \multicolumn{5}{|l|}{ Abnormal ECG } \\
\hline ST-T changes & - & $9(45.0 \%)$ & 0 & $0.002^{*}$ \\
\hline AVB & - & $8(40.0 \%)$ & $2(18.2 \%)$ & 0.20 \\
\hline IVCB & - & $5(25.0 \%)$ & $2(18.2 \%)$ & 0.66 \\
\hline Tachycardia & - & $3(15.0 \%)$ & $1(9.1 \%)$ & 0.63 \\
\hline APB & - & $1(5.0 \%)$ & $2(18.2 \%)$ & 0.25 \\
\hline VPB & & $5(25.0 \%)$ & $5(45.5 \%)$ & 0.25 \\
\hline
\end{tabular}




\begin{tabular}{lcccc} 
Abnormal Q & - & $2(10.0 \%)$ & $1(9.1 \%)$ & 0.93 \\
Sinus bradycardia & - & $2(10.0 \%)$ & 0 & 0.18 \\
\hline
\end{tabular}

1 


\section{Table 2 (on next page)}

\section{The CMR findings in AM, CM and NC groups}

Values are presented as mean \pm SE. $P 1$ : values comparison between AM and NC; $P 2$ : values comparison between CM and NC; P3: values comparison between AM and CM; ${ }^{*} P$ values adjusted for: None; ${ }^{\dagger} P$ values adjusted for: BSA and heart rate; ${ }^{\ddagger} P$ values adjusted for: BSA, heart rate, hematocrit and sex .

NC, normal control; AM, acute myocarditis; CM, subacute/chronic myocarditis; HLA, horizontal long axis; SA, short axis; ECV, extracellular volume; EDV, end-diastolic volume; ESV, end-systolic volume; LVM, LV mass; $\mathrm{SV}$, stroke volume; LVEF, left ventricle ejection fraction. 


\begin{tabular}{lllll}
\hline Groups & $\mathrm{NC}(\mathrm{n}=15)$ & $\mathrm{AM}(\mathrm{n}=20)$ & $\mathrm{CM}(\mathrm{n}=11)$ & $P$-values
\end{tabular}

Standardized cardiac morphology and function

$\begin{array}{lllll}\operatorname{EDV}(\mathrm{mm}) & 76.8 \pm 4.3 & 74.2 \pm 3.4 & 78.3 \pm 2.5 & 0.73 \\ \operatorname{ESV}(\mathrm{mm}) & 30.3 \pm 2.3 & 30.1 \pm 2.0 & 32.4 \pm 1.7 & 0.75 \\ \operatorname{LVM}\left(\mathrm{g} / \mathrm{mm}^{2}\right) & 48.8 \pm 2.5 & 47.8 \pm 1.9 & 44.6 \pm 2.7 & 0.48 \\ \mathrm{SV}\left(\mathrm{ml}^{-1}\right) & 46.6 \pm 2.4 & 44.2 \pm 1.6 & 45.9 \pm 2.4 & 0.68 \\ \operatorname{LVEF}(\%) & 61.1 \pm 1.3 & 60.1 \pm 1.1 & 58.6 \pm 2.0 & 0.51\end{array}$

Myocardial tissue characterization

$\begin{array}{lllll}\text { T2 }(\%) & - & 6(30.0 \%) & 2(18.2 \%) & 0.31 \\ \text { LGE }(\%) & - & 7(35.0 \%) & 4(36.4 \%) & 0.94\end{array}$

T1 and T2 values of "normal-appearing" myocardium $\quad \begin{array}{llll}P_{1} & P_{2} & P_{3}\end{array}$

$\begin{array}{lcccccc}\text { T2 SA, (ms) } & 37.2 \pm 0.3 & 37.4 \pm 0.5 & 37.1 \pm 0.2 & 0.54^{\ddagger} & 0.65^{\ddagger} & 0.09^{\ddagger} \\ \text { T1 }_{\text {SA }}(\mathrm{ms}) & 1297.6 \pm 8.0 & 1328.4 \pm 8.0 & 1320.8 \pm 13.0 & 0.09^{\ddagger} & 0.50^{\ddagger} & 0.38^{\ddagger} \\ \mathrm{ECV}_{\mathrm{SA}}(\%) & 27.0 \pm 0.6 & 28.1 \pm 0.5 & 30.1 \pm 0.9 & 0.20^{*} & 0.004^{*} & 0.57^{*} \\ \mathrm{~T}_{\text {HLA }}(\mathrm{ms}) & 37.1 \pm 0.5 & 36.7 \pm 0.4 & 37.2 \pm 0.5 & 0.08^{\ddagger} & 0.90^{\ddagger} & 0.60^{\ddagger} \\ \mathrm{T}_{\text {HLA }}(\mathrm{ms}) & 1323.3 \pm 8.6 & 1336.0 \pm 11.5 & 1305.1 \pm 8.0 & 0.56^{\ddagger} & 0.44^{\ddagger} & 0.05^{\ddagger} \\ \text { ECV }_{\text {HLA }}(\%) & 28.6 \pm 0.5 & 28.5 \pm 0.7 & 29.8 \pm 1.5 & 0.90^{*} & 0.35^{*} & 0.58^{*}\end{array}$




\section{Table 3 (on next page)}

The $\mathrm{T} 1$ and $\mathrm{T} 2$ values of "normal-appearing" myocardium in pediatric myocarditis patients with and without focal myocardial injuries on conventional MRI

Group I: patients with focal myocardial injuries;

Group II: patients without focal myocardial injuries.

Values are presented as mean $\pm \mathrm{SE} .{ }^{*} P$ values adjusted for: none; ${ }^{\dagger} P$ values adjusted for: $\mathrm{BSA}$, heart rate; ${ }^{\ddagger} P$ values adjusted for: $B S A$, heart rate and hematocrit; ${ }^{*} P$ values adjusted for: $B S A ;{ }^{~} P$ values adjusted for: $\mathrm{BSA}$, heart rate and sex; ${ }^{\sharp} P$ values adjusted for: $\mathrm{BSA}$, heart rate, hematocrit and sex.

ORs, odds ratios; $\mathrm{Cl}$, confidence interval; $\mathrm{HLA}$, horizontal long axis; SA, short axis; ECV, extracellular volume; BSA, body surface area. 


\begin{tabular}{|c|c|c|c|c|}
\hline & $\begin{array}{l}\text { Group I } \\
(n=11)\end{array}$ & $\begin{array}{l}\text { Group II } \\
(n=20)\end{array}$ & $\begin{array}{l}\text { Adjusted ORs } \\
(95 \% \mathrm{Cl})\end{array}$ & $P$ values \\
\hline $\mathrm{T} 2 \mathrm{sA}_{\mathrm{SA}}(\mathrm{ms})$ & $38.3 \pm 0.7$ & $36.6 \pm 0.3$ & $-1.0(-3.0,0.9)$ & $0.30^{\ddagger}$ \\
\hline Native T1 SA $(\mathrm{ms})$ & $1338.8 \pm 12.6$ & $1318.5 \pm 7.8$ & $-26.5(-52.5,-0.5)$ & $0.06^{* *}$ \\
\hline ECV ${ }_{S A}(\%)$ & $28.7 \pm 0.9$ & $28.9 \pm 0.6$ & $0.0(-2.1,2.1)$ & $0.99^{*}$ \\
\hline T2 HLA $(\mathrm{ms})$ & $37.2 \pm 0.6$ & $36.6 \pm 0.3$ & $0.1(-1.4,1.5)$ & 0.94 㧊 \\
\hline Native T1 HLA (ms) & $1332.1 \pm 15.9$ & $1323.6 \pm 8.8$ & $-3.1(-51.6,45.5)$ & $0.90^{\ddagger \ddagger}$ \\
\hline $\mathrm{ECV}_{\text {HLA }}(\%)$ & $28.4 \pm 1.2$ & $29.1 \pm 0.7$ & $1.2(-2.5,4.8)$ & $0.55^{*}$ \\
\hline
\end{tabular}




\section{Table 4(on next page)}

The associations between ECV and cardiac function in subacute/chronic myocarditis patients

*P values adjusted for: age, sex, heart rate and hematocrit. ${ }^{\dagger} P$ values adjusted for: age and hematocrit.

ECV, extracellular volume; LV, left ventricular; CM, subacute/chronic myocarditis; ORs, odds ratios; Cl, confidence interval; EDV, end-diastolic volume; ESV, end-systolic volume; LVM, left ventricular mass; SV, stroke volume; LVEF, left ventricle ejection fraction. 


\begin{tabular}{|c|c|c|}
\hline & Adjusted ORs(95\% Cl) & $P$ Value \\
\hline EDV & $-0.1(-0.2,0.1)$ & $0.30^{*}$ \\
\hline ESV & $-0.1(-0.4,0.3)$ & $0.78^{*}$ \\
\hline SV & $-0.2(-0.3,0.0)$ & $0.15^{*}$ \\
\hline LVM & $-0.1(-0.2,0.1)$ & $0.44^{\dagger}$ \\
\hline LVEF & $-0.4(-0.7,-0.1)$ & $0.03^{*}$ \\
\hline
\end{tabular}

2 